\title{
CONCEPT OF THE MAGNETIC LAUNCHER FOR MEDIUM CLASS UNMANNED AERIAL VEHICLES DESIGNED ON THE BASIS OF NUMERICAL CALCULATIONS
}

\author{
Miroseaw Kondratiuk, Leszek Ambroziak \\ Faculty of Mechanical Engineering, Bialystok University of Technology (BUT), Bialystok, Poland \\ e-mail:m.kondratiuk@pb.edu.pl; l.ambroziak@pb.edu.pl
}

\begin{abstract}
The paper presents a concept of a magnetic coil launcher for unmanned aerial vehicles of mass up to $25 \mathrm{~kg}$. The idea is not new, nevertheless in the paper, an innovative application of magnetic launcher technology for selected class of unmanned aerial vehicles is presented. So far, at Bialystok University of Technology, a magnetic coil launcher for micro aerial vehicles of mass up to $2.5 \mathrm{~kg}$ has been investigated. In the article, simulations of a conceptual multi-coil launcher with a magnetic core system are presented. The finite element method has been used in calculations. Moreover, in the paper, the concept of a magnetic support for transmission of mechanical power from the magnetic core to the launched payload is proposed. The applied methodology, computational results and potential technical difficulties of practical applications are also widely discussed.
\end{abstract}

Keywords: electromagnetic launcher, EML, magnetic support, permanent magnet, FEM

\section{Introduction}

Fast development and constantly increasing industrial applicability of unmanned aerial vehicles (UAVs) require new solutions as far as their operation systems are concerned. For instance, modern engineering associated with UAVs focuses especially on developing innovative navigation systems (Gosiewski et al., 2011; Kownacki, 2013), robust control of flight (Mystkowski, 2014), reliable security solutions, efficient electrical engines and power cells, formation of flight control algorithms (Gosiewski and Ambroziak, 2012), adaptive aerodynamics structures (Mystkowski, 2013), applications of so-called intelligent materials, etc. Generally speaking, most works are being carried out in order to increase the level of UAVs autonomy. There is also a major need for applying systems of assisted take-off and landing.

Starting launchers are used for many classes of UAVs from micro planes of mass up to a few kilograms to large military drones. Systems assisting take-off procedures increase the level of operators' safety, ensure recurrence of starts, decrease periods of time between consecutive starts, protect onboard equipment and UAV construction from undesired vibration and acceleration pulses. Employment of automatic launchers for UAV may also considerably increase the level of their autonomy and gives new possible applications, for example to autonomous systems for monitoring of countries boarders as well as unmanned systems of forest fire protection or unmanned aerial post services.

Presently, many different constructions are used as launching devices. Main solutions involve devices based on pneumatic (Perkowski, 2008) and hydraulic technology or are equipped with rubber or steam drive systems. Since the early 50's, advanced research on electromagnetic launchers (EML) have been conducted in the world. In some publications, the knowledge of magnetic launchers was clearly systematised, e.g. Kolm et al. (1980). Electromagnetic launchers are a completely different group of starting devices basing on conversion of stored electrical 
energy into kinetic energy of the launched object. Generally, there are two main types of EMLs: rail launchers and coil launchers. Moreover, coil launchers can be divided into synchronous and asynchronous (inductive) devices. More specified nomenclature and theory were presented in the following publications by Kondratiuk (2013), Tomczuk and Waindok (2009), Tomczuk et al. (2012). The idea of application of magnetic technology for aircraft launch is not new. Interesting papers concerning such topics have been already published (Patterson et al., 2002; McNab, 2007). Among others, very innovative research was carried out on electromagnetic launch systems for large airplanes. The investigations were conducted in the frame of big European project called GABRIEL (Sibilski et al., 2014). GABRIEL's research team proposed a system based on phenomena of superconducting levitation. As a result, the system can be capable of launching airliners such as Airbus A320. In the frame of the project, a magnetic drive system has been developed. The investigations involved numerical models, vibration analysis, CAD and design optimization procedures. Moreover, successful laboratory tests of a small scale launch system have been carried out and the outcomes were published, see Ładyżyńska-Kozdraś etal (2014a,b). Consequently, system GABRIEL has been designed for a large class of planes - commercial airliners. The concept of an electromagnetic linear drive for launching a medium class of UAVs significantly differs from solutions proposed by GABRIEL's research team. Main differences involve the type of magnetic linear drives, scale of the device, target performance, field of applications and approach to design.

In this paper, investigations on a synchronous coil launcher are described. Such a device consists of several serially located driving coils (usually made of a copper isolated wire) and a cylindrical core moving inside those coils. The core may be made of a ferromagnetic material, or a permanent magnet may be used. Comparing to pneumatic and rubber catapults, the synchronous coil launcher has many advantages, for instance, a quick recharging process and readiness for another shot, control of the launching force, modular construction and lack of complicated constructional parts exposed to damage.

So far, at Bialystok University of Technology (BUT) the electromagnetic launcher for micro-unmanned aerial vehicles (MAVs) has been developed and investigated (Kondratiuk, 2013). The construction of this device consists of ten serially located copper coils with a ferromagnetic core placed inside. The controlled magnetic field of the solenoids affects the core, and, in that way, the magnetic driving force is produced. The core is connected by means of a diamagnetic pusher to the carriage to which the launched plane is attached. The whole system is controlled by the open-source Arduino MEGA platform (ATMEGA2560) with the implemented algorithm of feedback control of MAV's position and acceleration. The construction, conducted design works and control structure were widely described in the previous papers by Kondratiuk and Gosiewski (2013a,b, 2014).

The main goal of the present work is to investigate and test some technical concepts of a multi-coil EML for middle class of UAVs. As tools for the testing, numerical simulations have been applied. Moreover, the article involves modifications, adaptation and scaling procedures of the previously constructed coil EML in order to launch objects of mass up to $25 \mathrm{~kg}$.

\section{Main assumptions}

Among different types of UAVs, there are various requirements for start conditions. The most important one is connected with the initial speed which is necessary to generate a proper lift force. Because of different UAVs geometries (shape, wing configuration, wingspan, etc.) different initial speed values are required. For instance, $25 \mathrm{~kg}$ delta wing planes require much greater velocity (about $25 \mathrm{~m} / \mathrm{s}$ ) than $25 \mathrm{~kg}$ gliders (about $15 \mathrm{~m} / \mathrm{s}$ ). In the paper, the design of an EML for $25 \mathrm{~kg}$ UAVs which ensure starting speed on the level of at least $20 \mathrm{~m} / \mathrm{s}$ is described. That value has been chosen arbitrarily as the goal to achieve. 
Another aspect connected with UAVs catapults concerns the source of power. In pneumatic and hydraulic launchers, the working fluid is accumulated under pressure in special tanks or containers. On the other hand, rubber launchers require an external force to stretch the elastic material while steam catapults need a whole complex system for preparing vapour of proper parameters. In EMLs, electrical energy should be accumulated in a suitably large storage from which it can be rapidly released by the launcher driving system. Previous investigations clearly showed that the capacitor bank perfectly meets this requirement.

In the proposed EML for $25 \mathrm{~kg}$ UAVs there can appear the necessity of switching very high currents flowing through driving coils (even up to $200 \mathrm{~A}$ ). From the practical point of view, for current control, IGBT transistors can be used in electronic switching circuits. During FEM simulations, data sheets of such electronic devices as transistors and capacitors are strictly taken into account.

Launchers for UAVs should also ensure sufficient stability, vibration and a safety level under take-off circumstances. These factors should be taken into account during design procedures.

One of the main assumptions connected with the coil EML for $25 \mathrm{~kg}$ UAVs is that initial investigations will be carried out on the already existing EML model for micro UAVs. Thus, this paper starts from possible modifications of the construction described in the $\mathrm{PhD}$ Thesis by Kondratiuk (2013).

\section{Model of the coil-core system}

The first possible modification is connected with the application of a permanent magnet as a driving core instead of the ferromagnetic one. Force characteristics of the coil-core system with the ferromagnetic and with the magnetic cores were computed by means of the finite element method, (Tomczuk et al., 2007). COMSOL Multiphysics software is employed as the ready-made computer program for electromagnetic computations. In the FEM model of the coil-core system, the advantage of the axial symmetry is used. The partial differential equation (PDE) describing the distribution of static magnetic field in the coil-core system is called Ampère's law, and in the ferromagnetic core domain it takes the following form

$$
\nabla \times\left(\frac{\nabla \times \mathbf{A}}{\mu_{0} \mu_{r}(|\mathbf{B}|)}\right)=\mathbf{J}_{e}
$$

where $\nabla$ denotes the nabla operator; $\mathbf{A}$ - magnetic vector potential, $[\mathrm{Wb} / \mathrm{m}] ; \mu_{0}=4 \pi \cdot 10^{-7} \mathrm{H} / \mathrm{m}$ - permeability of vacuum; $\mu_{r}(|\mathbf{B}|)$ - relative permeability of a nonlinear ferromagnetic material, [dimensionless]; $|\mathbf{B}|$ - magnetic flux density, $[\mathrm{T}] ; \mathbf{J}_{e}$ - external current density, $\left[\mathrm{A} / \mathrm{m}^{2}\right]$.

In the cylindrical coordinate system, the vector $\mathbf{A}$ can be written as

$$
\mathbf{A}=A_{r} \widehat{\mathbf{r}}+A_{\varphi} \widehat{\varphi}+A_{z} \widehat{\mathbf{z}}
$$

where $A_{r}, A_{\varphi}, A_{z}$ are components of the vector $\mathbf{A},[\mathrm{Wb} / \mathrm{m}] ; \widehat{\mathbf{r}}, \widehat{\boldsymbol{\varphi}}, \widehat{\mathbf{z}}-$ unit vectors.

Thus, rotation of $\mathbf{A}$ is equal

$$
\nabla \times \mathbf{A}=\left(\frac{1}{r} \frac{\partial A_{z}}{\partial \varphi}-\frac{\partial A_{\varphi}}{\partial z}\right) \widehat{\mathbf{r}}+\left(\frac{\partial A_{r}}{\partial z}-\frac{\partial A_{z}}{\partial r}\right) \widehat{\varphi}+\frac{1}{r}\left(\frac{\partial\left(r A_{\varphi}\right)}{\partial r}-\frac{\partial A_{r}}{\partial \varphi}\right) \widehat{\mathbf{z}}
$$

Ampère's law for the magnet domain has a different form than (3.1)

$$
\nabla \times\left(\frac{\nabla \times \mathbf{A}}{\mu_{0} \mu_{r}}-\mathbf{B}_{r}\right)=\mathbf{J}_{e}
$$

where $\mathbf{B}_{r}$ is the magnet remanent flux density vector, $[\mathrm{T}]$. 
Coil and ferromagnetic core parameters used in the simulation are taken from the real EML model previously described by Kondratiuk (2013), Kondratiuk and Gosiewski (2013a,b, 2014). The catalogue value of remanent magnetic flux density of the simulated magnet is equal to $1.24 \mathrm{~T}$. Relative permeability of the magnet material is equal to $\mu_{r} \approx 1.05$, but it can be modelled as close to 1 because, in order to generate a magnetic force, the magnet should be remagnetized so the external magnetic field produced by the coils increases magnetic induction in the magnet according to the following constitutive relation

$$
\mathbf{B}=\mu_{0} \mu_{r} \mathbf{H}+\mathbf{B}_{r}
$$

where $\mathbf{H}$ is the magnetic field vector, $[\mathrm{A} / \mathrm{m}]$.

Differences in the values of magnetic flux density for $\mu_{r}=1$ and $\mu_{r}=1.05$ obtained in a freely chosen point located inside the magnet are shown in Fig. 1.

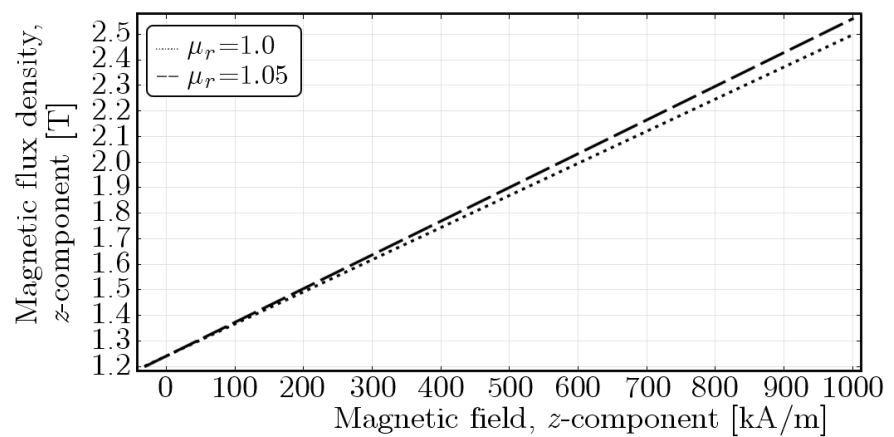

Fig. 1. Magnetic flux density in the magnet material during remagnetization

In fact, relative permeability of a permanent magnet decreases under influence of very strong external field and finally achieves the value of 1. Then, both lines in Fig. 1 line up parallelly. In the model, this effect is neglected.

A permanent magnet has been proposed instead of a ferromagnetic one in order to increase the magnetic force. In the FEM model, the Maxwell surface stress tensor method (MSST) and the virtual work (VW) method have been tested. Generally, both methods give similar results and they are applied alternatively. In order to present the advantage of application of a permanent magnet, the computed magnetic forces acting on both cores (magnetic and ferromagnetic) under influence of coil current $i_{c}=1 \mathrm{~A}$ and $i_{c}=3 \mathrm{~A}$ are compared in Fig. 2.

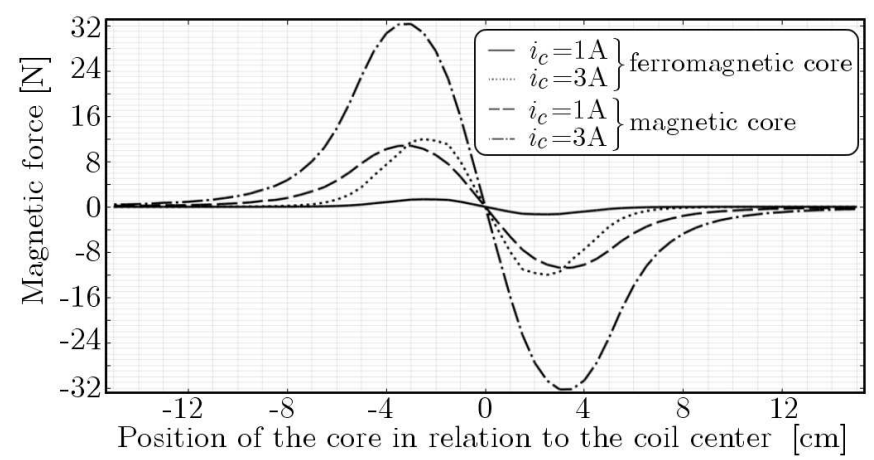

Fig. 2. Comparison of magnetic forces acting on the ferromagnetic and magnetic core

The calculations clearly show that the magnetic core can provide much greater magnetic force than the ferromagnetic core. The computed results for the ferromagnetic core have been experimentally verified and high level data similarity has been revealed (Kondratiuk, 2013). 
In Fig. 3, 3D and 2D views of the modelled coil-magnet system are presented. Both representations are developed from the $2 \mathrm{D}$ axi-symmetric model by $2 \mathrm{D}$ revolution and $2 \mathrm{D}$ mirror functions respectively applied to the FEM solution.
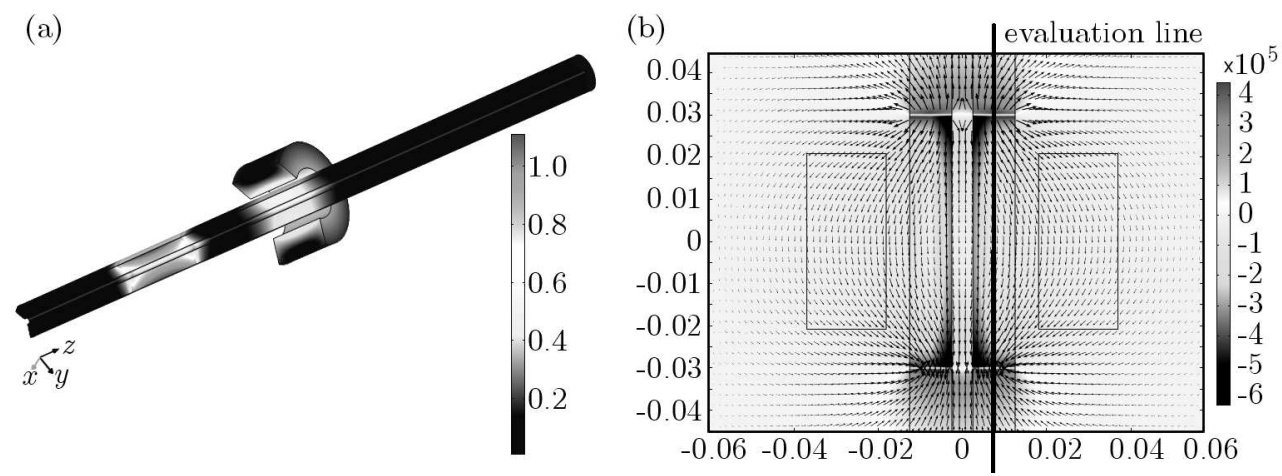

Fig. 3. Development of the FEM axi-symmetric solution for the coil-magnet system into 3D and 2D views: (a) magnetic flux density for the system under coil current $i_{c}=10 \mathrm{~A}$ and with the magnet position $z_{m}=-12 \mathrm{~cm}$, (b) magnetic field distribution (z-coordinate) around the system without current and with the magnet inside

In order to model motions of the core along the coil $z$-axis the Euler method has been applied (Kondratiuk, 2013). In the FEM model, a function of core length has been introduced. That function multiplied by remanent magnetic flux density, magnet permeability or conductivity describes properties of the core along the $z$-coordinate. In the model, isotropy of the above mentioned parameters has been assumed. In Fig. 3b, the evaluation line is drawn. Along this line, some crucial model parameters have been calculated and the results are presented in Fig. 4.

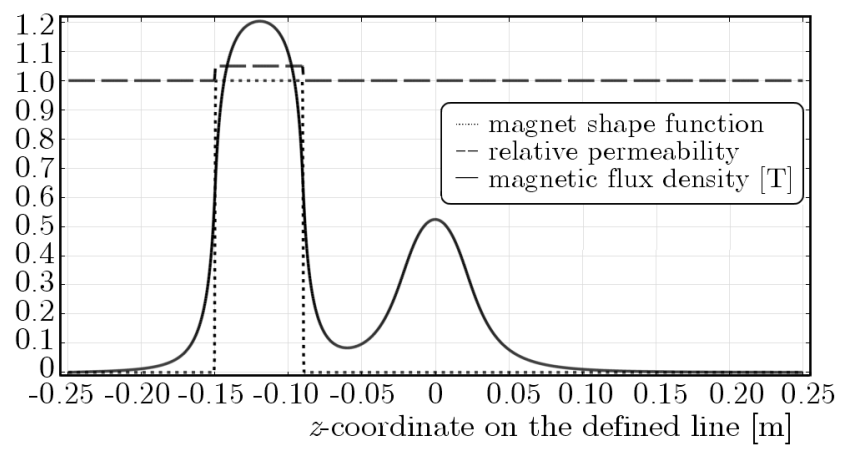

Fig. 4. Distribution of crucial parameters of the model along a selected line in the $z$-coordinate (coil current $i_{c}=20 \mathrm{~A}$, coil position $p_{c}=0$, magnet position $z_{m}=-12 \mathrm{~cm}$ )

Positions of the core/magnet $\left(z_{m}\right)$ or the coil $\left(p_{c}\right)$ are defined as locations of their centres in the global coordinate system. The magnetic flux density distribution presented in Fig. 4 comes from the magnet (higher pick) and from the coil (lower pick). This also explains from where the magnetic force comes from. The magnetic field tries to retain uniformity and always acts in the opposite way to any changes, for example in the coil current value or in the system geometry. When the coil generates an external field, the magnet and solenoid are attracted to each other because only in that way the total magnetic field can become more uniform.

\section{Multi-coil EML model}

The model of a multi-coil EML can be divided into two strongly related parts: electromagnetic and mechanical. 


\subsection{Electromagnetic part of the multi-coil EML}

The first model of EML with a permanent magnet as the core is based on the previously constructed and widely investigated 10-coil EML with a ferromagnetic core. Parameters of that construction were presented in previous publications by Kondratiuk (2013), Kondratiuk and Gosiewski (2013a,b, 2014). It is worth noticing that the investigated coils were made of a $0.8 \mathrm{~mm}$ isolated copper wire in configuration 27 per 52 turns (1391 turns in total). In the paper, a code for coils description was proposed. For instance, the aforementioned solenoid can be coded as $0.8 \times 27 \times 52$.

Each coil in the model affects the magnetic core through magnetic field. Strength and distribution of the field depend on coils configuration and currents intensity flowing through the wires. However, the magnetic force is directly proportional to the current value, so in order to simplify the model, it is possible to compute the magnetic force distribution for different core positions in relation to the coil centre and only for one current value $i_{c}=1 \mathrm{~A}$. It incorporates the function $F\left(1 \mathrm{~A},\left[z_{m}-p_{n}\right]\right)$ in the multi-coil EML model as follows

$$
F_{n}\left(i_{n}, z_{m}\right)=i_{n} F\left(1 \mathrm{~A},\left[z_{m}-p_{n}\right]\right)
$$

where $F_{n}\left(i_{n}, z_{m}\right)$ is the magnetic force generated by the $n$-th coil, $[\mathrm{N}] ; i_{n}$ - coil current flowing through the $n$-th coil, [A]; $p_{n}-n$-th coil position, [m].

The total force acting on the magnet can be defined as

$$
F_{m}=\sum_{n=1}^{N} F_{n}\left(i_{n}, z_{m}\right)
$$

where $N$ is the number of coils located serially.

As the core, an assembly of 6 ring-shaped magnets of remanence $B_{r}=1.24$ T made of material N38 (Arnold, 2014) is proposed. Dimensions of selected magnets are the following: $27 \mathrm{~mm}$ - outer diameter, $5 \mathrm{~mm}$ - inner diameter, $10 \mathrm{~mm}$ - magnet height. Each magnet is magnetized along $10 \mathrm{~mm}$ dimension. The assembled core is $60 \mathrm{~mm}$ long. The number and shape of magnets are selected arbitrarily. Similarly to the coils, in the paper, a code for core description is proposed. The above described core can be coded as $6 \times$ RSM- $27 \times 5 \times 10-\mathrm{N} 38$ where RSM means a ringshaped magnet. Visualization of the proposed core in front of two coils $0.8 \times 27 \times 52$ is presented in Fig. 5.

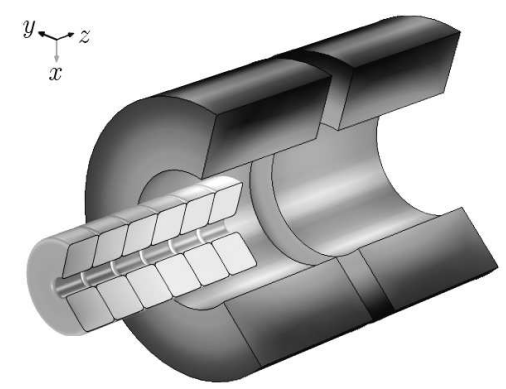

Fig. 5. Cross-sectional visualization of the core $6 \times$ RSM-27 $\times 5-10-N 38$ in front of the two coils $0.8 \times 27 \times 52$

Stationary calculations for the coil $0.8 \times 27 \times 52$ and the assembled magnetic core $6 \times$ RSM$-27 \times 5 \times 10-\mathrm{N} 38$ have been carried out and the function $F\left(1 \mathrm{~A},\left[z_{m}-p_{n}\right]\right)$ calculated (Fig. 6$)$. The function presented in Fig. 6 has been used according to equation (4.1) in the 10-coil EML model.

Regarding the power source in the model, a single bank of capacitors connected parallelly has been applied. First calculations have been conducted for the system voltage of $340 \mathrm{~V}$ and the 


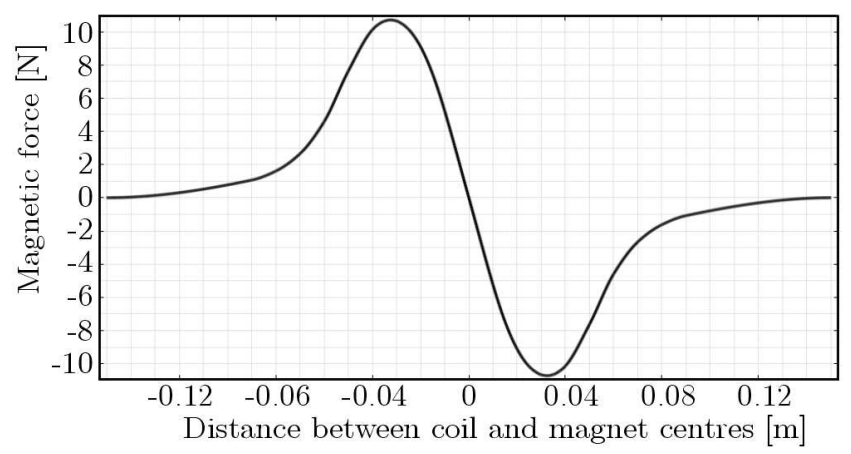

Fig. 6. Function $F\left(1 \mathrm{~A},\left[z_{m}-p_{n}\right]\right)$ calculated for $p_{n}=0$

total capacity of $94 \mathrm{mF}$. In the simulation, the capacitor discharging process has been modelled according to the following equations

$$
u_{c}(t)=u_{c 0}-\frac{1}{C} \int_{0}^{t} i_{c}(t) d t \quad i_{c}(t)=\sum_{n=1}^{N} i_{n}(t)
$$

where $u_{c}(t)$ is capacitor voltage, $[\mathrm{V}] ; u_{c 0}$ - initial capacitor voltage, $[\mathrm{V}] ; C$ - capacity of the bank, $[\mathrm{F}] ; i_{c}(t)$ - total capacitor discharging current, $[\mathrm{A}] ; i_{n}(t)$ - current flowing through $n$-th coil, $[\mathrm{A}]$.

\subsection{Mechanical part of the multi-coil EML}

In the mechanical part of the model, the damping force $\left(F_{d}\right)$ is introduced

$$
F_{d}\left(v_{m}\right)=\underbrace{b_{d} v_{m}}_{\text {holds always }} \quad \text { or } \quad F_{d}\left(v_{p}\right)=\underbrace{b_{d} v_{p}}_{\begin{array}{c}
\text { holds during } \\
\text { acceleration } \\
\left(v_{m}=v_{p}\right)
\end{array}}
$$

where $b_{d}$ denoted the damping coefficient, $[\mathrm{N} \cdot \mathrm{s} / \mathrm{m}] ; v_{m}, v_{p}$ - magnet and payload (UAV) velocities, respectively, $[\mathrm{m} / \mathrm{s}]$.

A simplified mechanical scheme of the system is presented in Fig. 7.

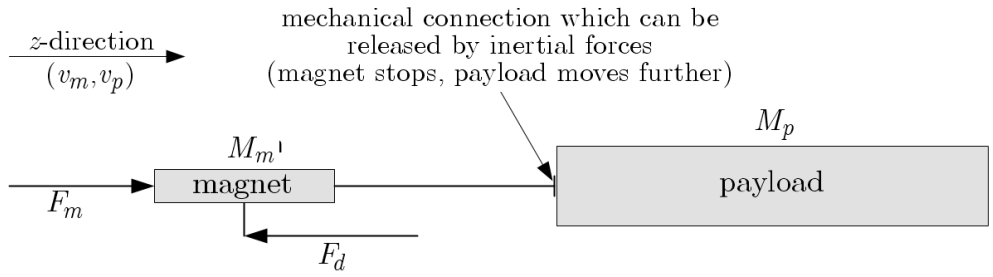

Fig. 7. Mechanical scheme of the investigated system

During simulations, the damping coefficient value has been arbitrarily chosen as constant $b_{d}=5 \mathrm{~N} \cdot \mathrm{s} / \mathrm{m}$. Dynamic behaviour of the multi-coil EML with magnetic core and a $25 \mathrm{~kg}$ payload (launching UAV) is described by the following equations:

- for $z_{p}<$ braking coil centre

$$
\frac{d^{2} z_{m}}{d t^{2}}=\frac{d^{2} z_{p}}{d t^{2}}=\frac{F_{m}-F_{d}\left(v_{m}\right)}{M_{m}+M_{p}}
$$


— for $z_{p} \geqslant$ braking coil centre

$$
\frac{d^{2} z_{m}}{d t^{2}}=\frac{F_{m}-F_{d}\left(v_{m}\right)}{M_{m}} \quad \frac{d^{2} z_{p}}{d t^{2}}=0
$$

where $z_{m}, z_{p}$ are magnet and payload positions, [m]; $M_{m}, M_{p}$ - magnet and payload masses, [kg]; $F_{m}$ - magnetic force, [N].

Equations (4.5) and (4.6) mean that during the launch phase both magnet and UAV masses are accelerated. When the acceleration changes its sign to minus, the braking phase starts. The UAV disconnects from the magnet and after that, only the magnetic core mass takes part in the braking process (no acceleration acts on the payload). Mass of the core $6 \times \mathrm{RSM}-27 \times 5 \times 10-\mathrm{N} 38$ is equal to $0.25 \mathrm{~kg}$, but in the model $M_{m}=1 \mathrm{~kg}$. In that way, any masses contained in necessary constructional pushers, screws, fixings, etc., are taken into account.

\subsection{Characteristics of the non-modified multi-coil EML model}

In this Subsection, the results of simulations conducted on the non-modified multi-coil EML are presented. 'Non-modified' means that the calculations have been carried out for a construction consisting of 10 serially located coils $0.8 \times 27 \times 52$ designed for micro UAVs and widely described by Kondratiuk (2013), Kondratiuk and Gosiewski, 2013a,b, 2014). The core $6 \times$ RSM$27 \times 5 \times 10$-N38 has been applied. In Fig. 8 , the power source characteristics come from simulation of the EML with the magnetic core and launching a $25 \mathrm{~kg}$ UAV are presented.

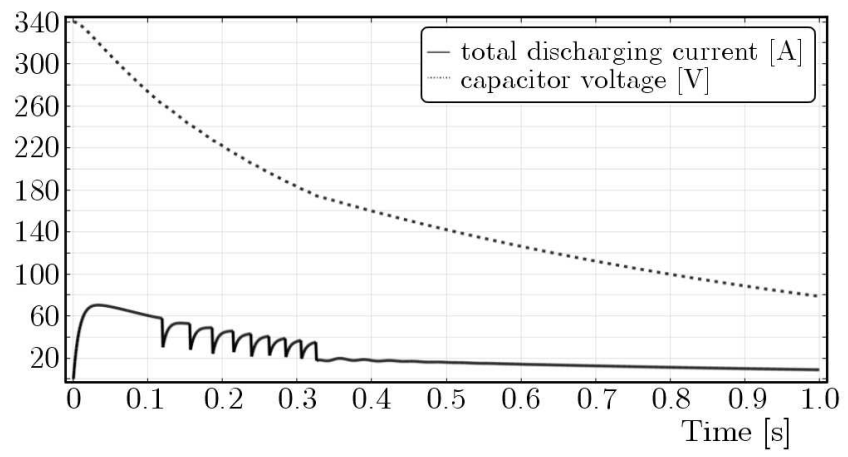

Fig. 8. Characteristic of the capacitors bank voltage $(340 \mathrm{~V} / 94 \mathrm{mF})$ and characteristic of the total discharging current from simulation of the launch of a $25 \mathrm{~kg}$ UAV by means of the EML with magnet core

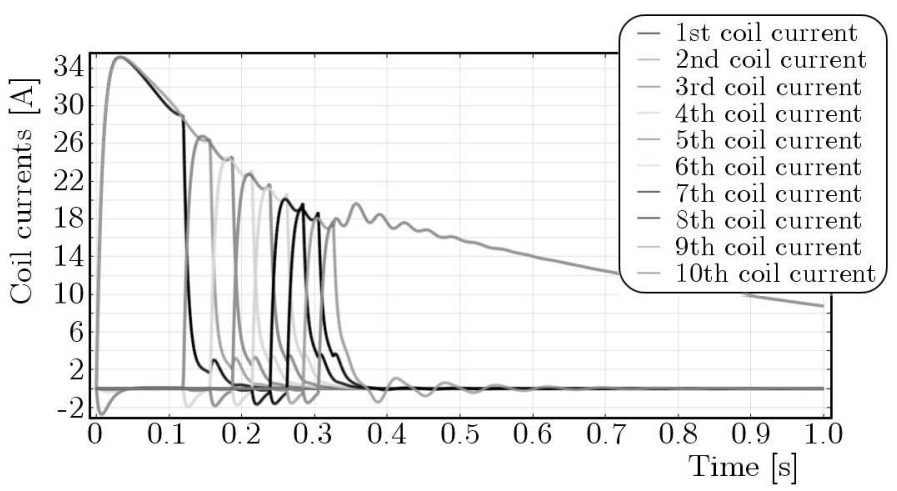

Fig. 9. Currents flowing through particular coils of the EML during simulation of the $25 \mathrm{~kg}$ UAV launch

In Fig. 9, the time evolutions of coil currents are presented. The last coil works until the magnetic core stops in its centre. The characteristics show that in the model electromagnetic 
induction is taken into account. Changing currents in particular coils generate electromotive force in the neighbouring solenoids.

In the model, current can flow through wires in both directions. In reality it can be compensated by introducing switching transistors (IGBT) and safety rectifying diodes into control electronic systems. In the model, a semi-coil system has been applied. Two neighbouring coils are powered on simultaneously. When the core achieves the centre of the first coil, it is turned off and the next is powered on. This method is directly visible on the current characteristics in Fig. 9. Moreover, an influence of the capacitor power source can also be noticed because the maximum amplitude of currents decreases with time like as the capacitor voltage. This effect is caused by the finite capacity introduced into the model and directly shows how the power source parameters are significant.

In Fig. 10, characteristics of the magnetic force acting on the moving system of the magnet with payload are presented. The last coil can be recognized as a magnetic brake. Moreover, the damping force $\left(F_{d}\right)$ value is also shown. That force can reach considerable values for higher velocities.

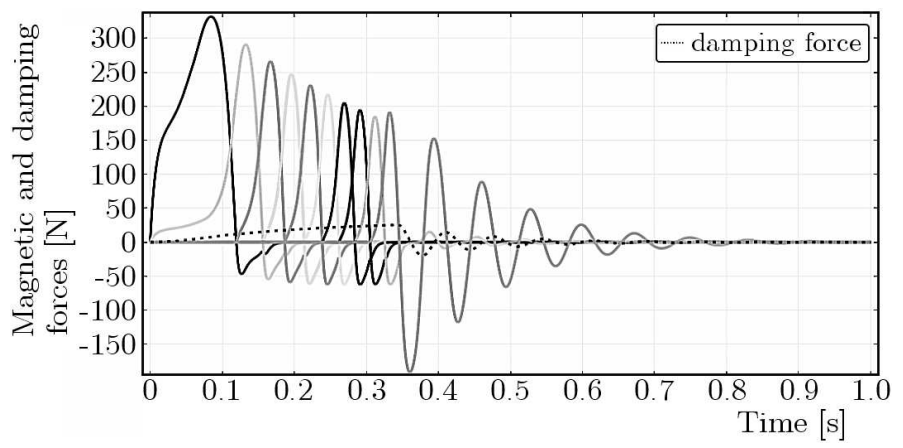

Fig. 10. Forces generated by particular coils of the EML during simulation of the $25 \mathrm{~kg}$ UAV launch

In Fig. 11, velocity characteristics are presented. The simulation reveal that the construction of 10 serially located coils $0.8 \times 27 \times 52$ with the core $6 \times \mathrm{RSM}-27 \times 5 \times 10-\mathrm{N} 38$ can ensure a $25 \mathrm{~kg}$ UAV only a $2.5 \mathrm{~m} / \mathrm{s}$ initial speed. It is less by an order than the assumed $20 \mathrm{~m} / \mathrm{s}$.

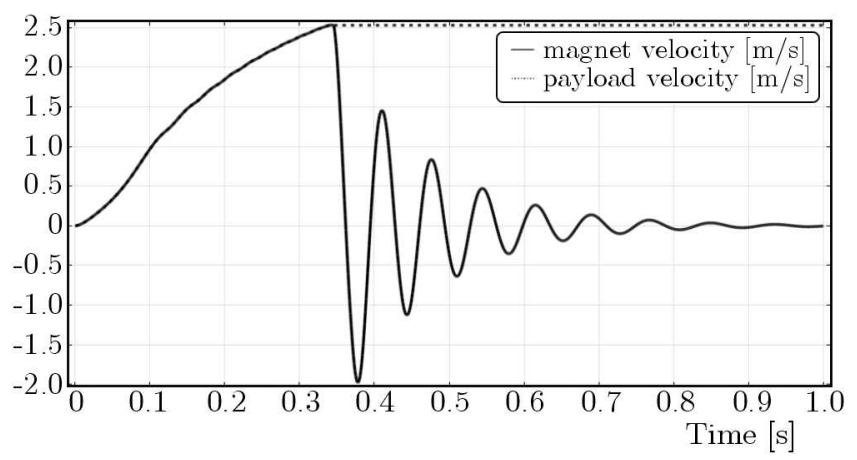

Fig. 11. Velocity characteristics from simulation of the $25 \mathrm{~kg}$ UAV launch

\section{Modification of the EML}

The computed results presented in the previous Section indicate that in order to increase the starting velocity, some modifications in the structure of the investigated EML should be introduced. 


\subsection{EML consisting of 4 parallel 20 -coil $(0.8 \times 27 \times 52)$ modules}

As the first natural improvement, an increase in the coil number has been proposed (up to 20). Another modification is based on the parallel connection of a few of 20-coil modules (mechanical and electrical connection). In the modified FEM model, a single capacitor bank is used as the power source for all coils and modules. Thus, the capacitor voltage is increased up to $1 \mathrm{kV}$. Four modules consisting of 20 coils $0.8 \times 27 \times 52$ and mechanically connected in parallel have been investigated. The magnetic force generated by the module and coil currents are totalized. The electromagnetic interaction between each module is neglected. The whole system of 4 driving modules is coupled by common dynamic equations (4.5) and (4.6).

In Fig. 12, simulation characteristics of the capacitor bank $(1 \mathrm{kV} / 94 \mathrm{mF})$ of four modules consisting of $200.8 \times 27 \times 52$ coils each and connected in parallel as shown. It is worth noticing that the maximum value of the total discarding current increased by factor of 2.5 .

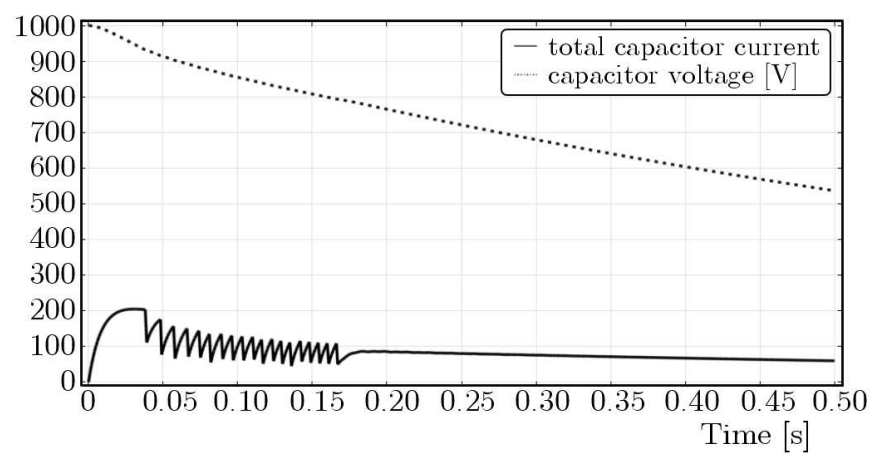

Fig. 12. Simulation characteristics of the capacitors bank $(1 \mathrm{kV} / 94 \mathrm{mF})$ of four modules consisting of 20 $0.8 \times 27 \times 52$ coils each connected in parallel

Velocity characteristics of the modified EML are presented in Fig. 13. Improvements introduced into the model give an increase in the starting velocity up to $8.5 \mathrm{~m} / \mathrm{s}$. It is still not enough. The decision of coils configuration and the core structure modification has been made.

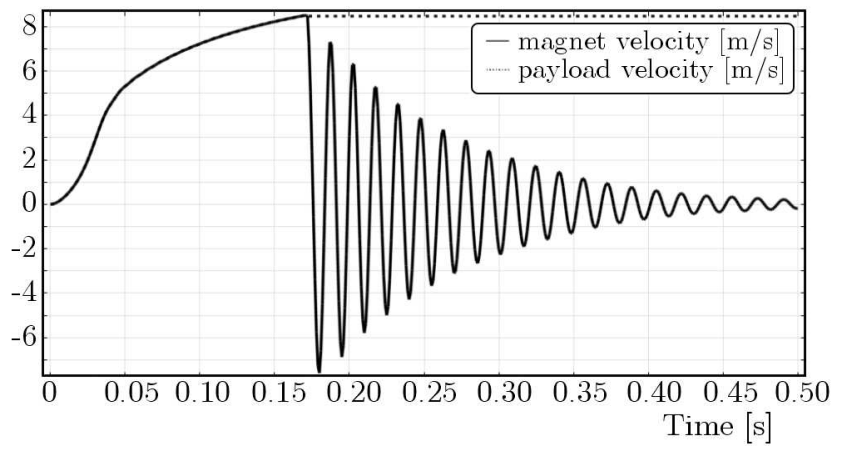

Fig. 13. Velocity characteristics from simulation of the launch of $25 \mathrm{~kg}$ UAV by means of the 4 EML module with magnet core connected in parallel

\subsection{EML consisting of 6 parallel 20 -coil $(1.0 \times 10 \times 150)$ modules and the core $17 \times$ RSM-28 $\times 10 \times 10-N 52$}

In order to achieve the previously assumed velocity of $20 \mathrm{~m} / \mathrm{s}$ a few modifications in the coils configuration and the core (magnet) structure have been proposed.

Firstly, the core $6 \times$ RSM- $27 \times 5 \times 10-\mathrm{N} 38$ has been replaced by $17 \times$ RSM- $28 \times 10 \times 10$-N 52 . It comprises 17 ring-shaped magnets made of material N52 (Arnold, 2014) and with remanence $B_{r}=1.45 \mathrm{~T}$. Secondly, the coils $0.8 \times 27 \times 57$ have been replaced by $1.0 \times 10 \times 150$ made of an 
isolated copper wire of $1 \mathrm{~mm}$ diameter. The distance between the coils is set to $1 \mathrm{~cm}$, thus the driving part of the EML is about $3.3 \mathrm{~m}$ in length. The core and coils configurations are chosen arbitrarily. Thirdly, the number of parallel modules is increased up to 6. Finally, the capacity of the electrical energy bank is changed to $825 \mathrm{mF}$. It is a high value but can be simply achieved by the parallel and serial connection of a few hundreds of electrolytic capacitors $(450 \mathrm{~V} / 3.3 \mathrm{mF})$. In the new model of the EML capacitor bank, the voltage is set to $1 \mathrm{kV}$. Initial simulations reveal that more than one coil should be used to stop the core. In the final model, the last three solenoids act as magnetic brakes.

In Fig. 14, simulation characteristics of the capacitor bank $(1 \mathrm{kV} / 825 \mathrm{mF})$ of six modules consisting of $201.0 \times 10 \times 150$ coils each and connected in parallel are presented. The maximum value of the total discarding current significantly has increased up to $2.2 \mathrm{kA}$. However, currents of that value flow only in a short period of time, so it can be practically applied in the real device. Moreover, the currents flowing out from the electrical energy bank are spread over all capacitors. The simulated currents flowing through particular coils are shown in Fig. 15. Their maximum values are not higher than $190 \mathrm{~A}$.

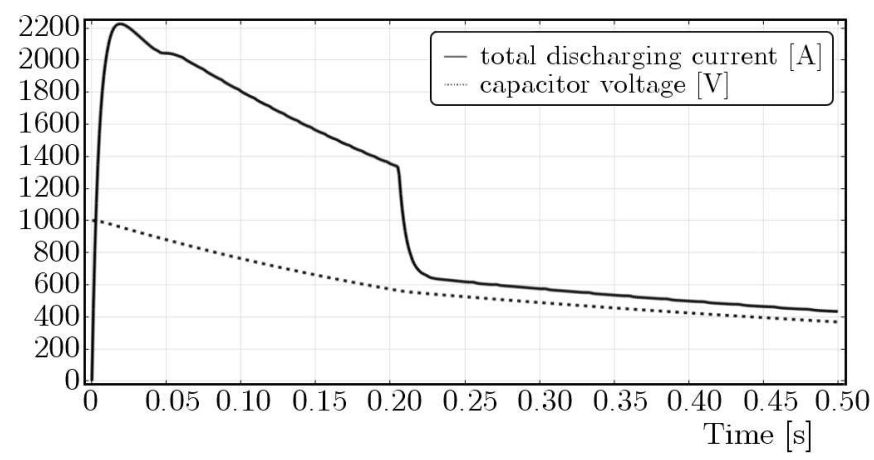

Fig. 14. Characteristics of the capacitor bank $(1 \mathrm{kV} / 825 \mathrm{mF})$ supplying six modules consisting of 20 $1.0 \times 10 \times 150$ coils each and connected in parallel

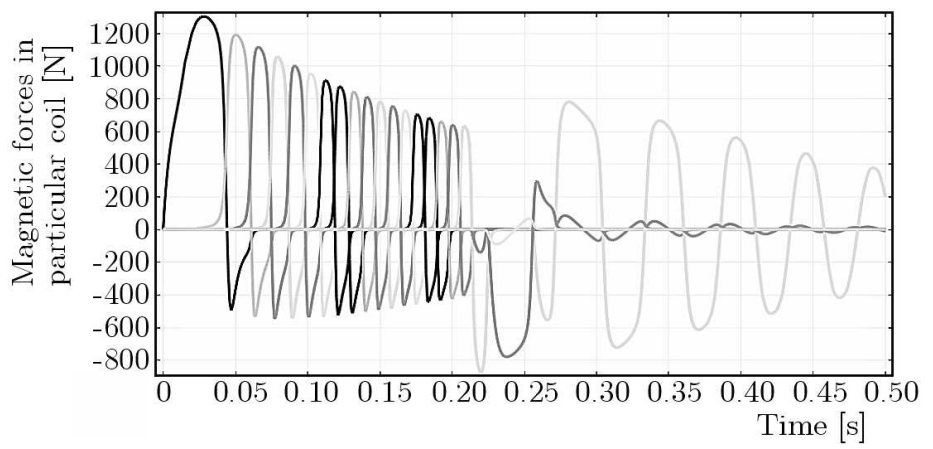

Fig. 15 . Forces generated by particular coils of the single EML module $(20$ coils $1.0 \times 10 \times 150)$

In Figs. 16 and 17, the force characteristics are presented. They clearly show that the last three coils act as a magnetic brake. Influence of the introduced damping force is also visible. In Fig. 18, the velocity characteristics are shown. The modification introduced into the EML model enables the starting velocity to achieve the previously assumed value of $20 \mathrm{~m} / \mathrm{s}$.

\section{Magnetic support}

In the paper, a project of the magnetic slideway track for launched objects is also proposed. During the start, the supporting UAV of mass up to $25 \mathrm{~kg}$ may be a challenging task because of 


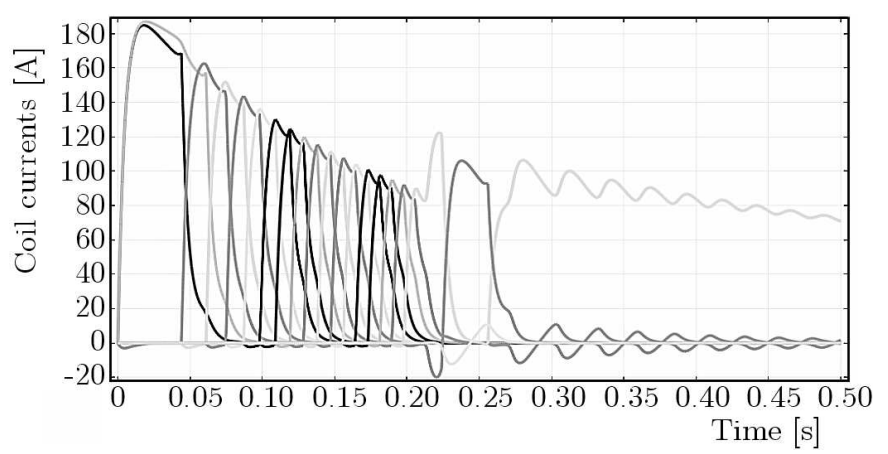

Fig. 16. Currents in particular coils of each module

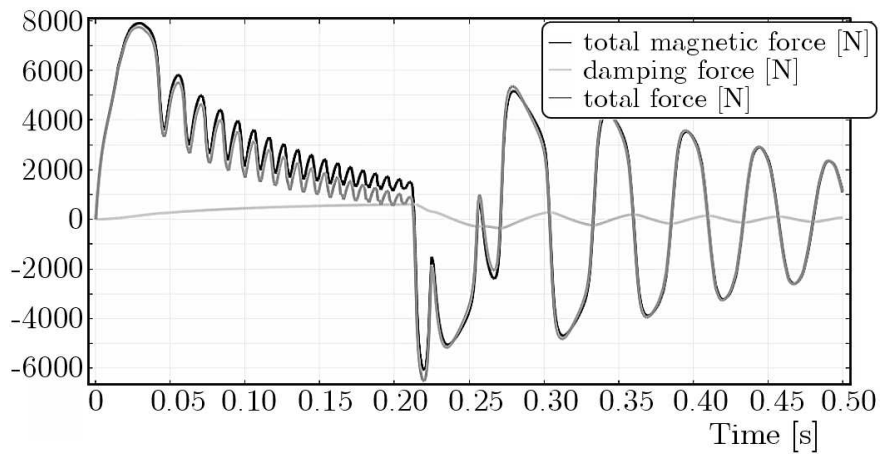

Fig. 17. Forces of the modified EML (6 modules)

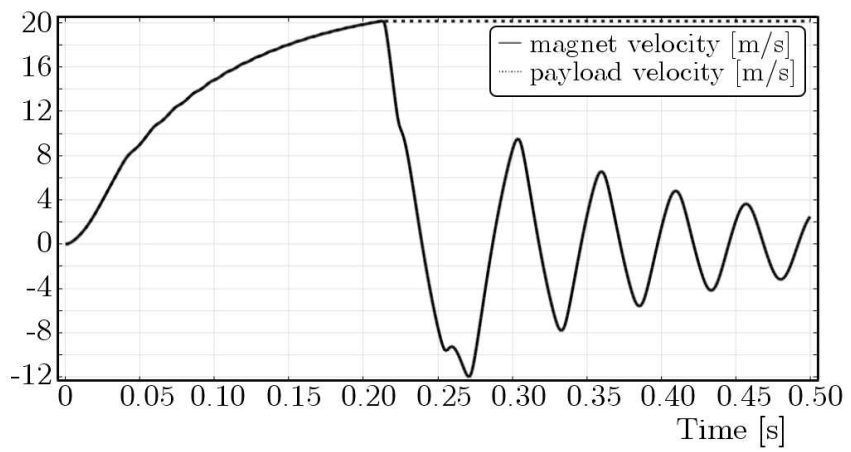

Fig. 18. Velocity characteristics of the modified EML (6 modules)

gravity, friction and material elastic buckling. The designed support solution consists of several permanent magnets of different sizes. The proposed solution creates a magnetic spring on which friction forces are minimized and the gravity is compensated for by magnetic repulsion. This system has also been modelled by means of FEM. The calculations have been conducted for different magnets and, as a result, the geometry configuration and magnet properties suitable for this particular type of support have been determined.

In Fig. 19, the concept of a magnetic track is presented. The construction consists of six downside (main) magnets BM- $80 \times 20 \times 10-\mathrm{N} 35 \mathrm{H}$ (BM - board magnets, length $[\mathrm{mm}] \times$ width $[\mathrm{mm}] \times$ height $[\mathrm{mm}], \mathrm{N} 35 \mathrm{H}-$ magnet material (Arnold, 2014)) and ten upside (stabilizing) magnets BM- $40 \times 10 \times 4$-N35. The carriage moving in the $x$-direction comprises four magnets $\mathrm{BM}-80 \times 20 \times 10-\mathrm{N} 35 \mathrm{H}$.

From the FEM models, the magnetic forces acting on the carriage consisting of 4-magnet have been computed. In Fig. 20, the force acting in the $z$-direction is presented as a function of the carriage position in the $z$-direction (zero position means contact between the carriage and 


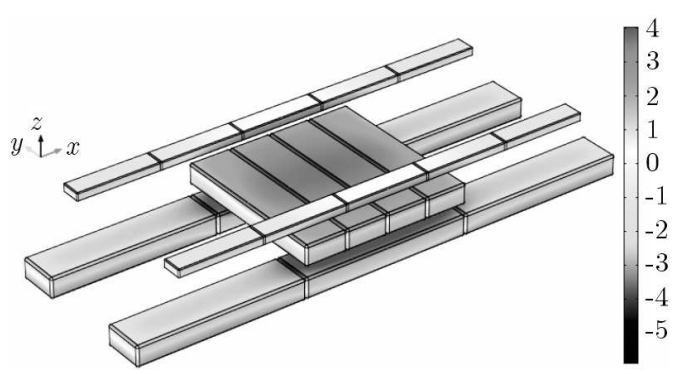

Fig. 19. The proposed magnetic track with the distribution of magnetic vector potential
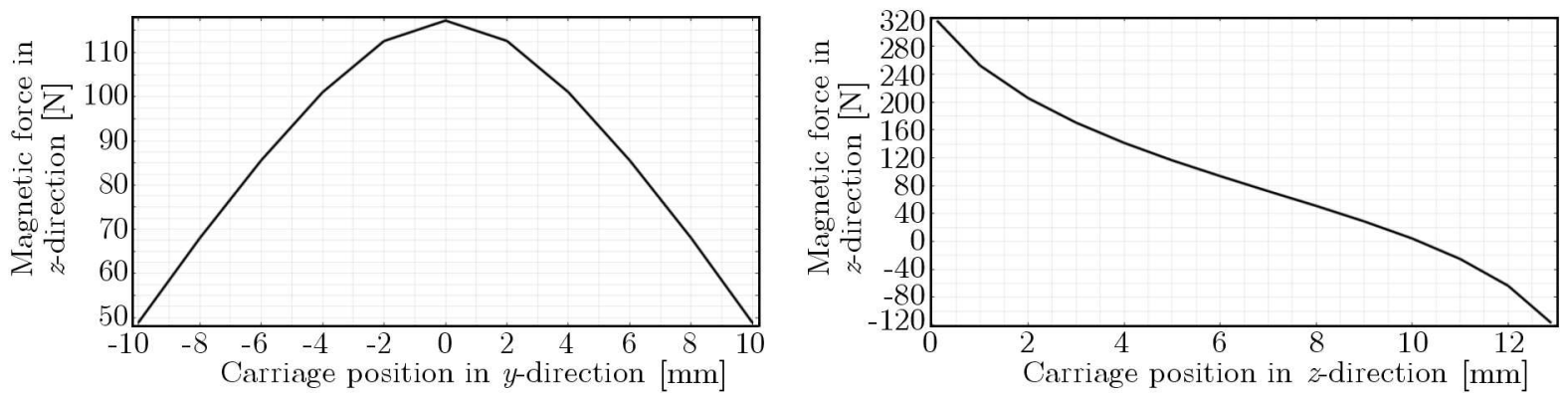

Fig. 20. Magnetic force acting on the 4-magnet carriage in the $z$-direction

downside magnets, $13 \mathrm{~mm}$ means contact with the upside magnets) and in the $y$-direction (zero means central location of the carriage).

In Fig. 21, the magnetic force acting on the 4-magnet carriage in the $y$-direction is presented as a function of the carriage position in the $y$-direction.

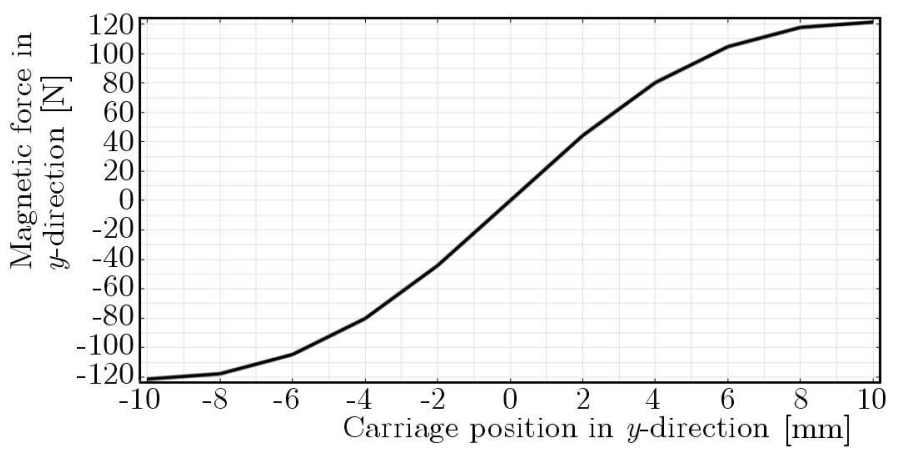

Fig. 21. Magnetic force acting on the 4-magnet carriage in the $y$-direction

The computed results are very optimistic and prove that the proposed magnetic support can be an applicable part in the real model of the coil EML for UAVs of mass up to $25 \mathrm{~kg}$. Nevertheless, the magnetic force is strongly dependent on the carriage position and may cause instability of the slideway. In real objects, a bearing system in the $y$-direction should be applied.

\section{Remarks and future works}

The nearest future works should be connected with experimental verification of the magnetic forces generated by the investigated coils and acting on the proposed magnetic core. The important part is also CAD design of the EML construction consisting of six driving modules (each having 20 coils). The problems of power transmission from the core to the carriage and 
the launched UAV should also be deeply investigated and solved. Moreover, other constructional aspects should be taken into design considerations, for instance, shape and materials of the frame, transport possibilities, system assembly and disassembly, power system connections, electronic control systems, safety conditions, fixing UAV on the launcher, mechanical strength of the crucial elements, etc. So far, the conducted research has revealed that the described project is quite challenging and very multidisciplinary. The obtained results and designed solutions are optimistic and give a real chance for practical realisation of the magnetic coil EML for UAVs of mass up to $25 \mathrm{~kg}$.

Works conducted so far on magnetic launchers, assisted start procedures and UAVs autonomy inspired authors to think on the design of a fully operational autonomous system for observation and inspection of extensive territories and regions such as forests, swamps, deserts or even border zones. The main assumption is that the proposed system should operate and perform its missions without any human assistance. Such a system should consist of an operational micro or medium class wing-plane adjusted to particular missions, coil magnetic launcher, landing subsystem, generator or battery used as the power source and control system. An airplane with wings can cover much longer distance than quadro-, hexa- or octocopters in the same amount of time and with lower energy cost. In order to carry out a mission, such an UAV can be launched in precisely defined periods of time, for example, minutes, hours or days. A hard and challenging task is to accomplish the UAV landing process after which the airplane should almost instantly be prepared for another launch without any human assistance. In our opinion, development of the landing subsystem is crucial for practical application of the proposed autonomous system. First conceptual ideas have been already born and they will be investigated in the near future.

Acknowledgement

The study has been financed by Bialystok University of Technology as the own research work $\mathrm{MB} / \mathrm{WM} / 11 / 2013$.

\section{References}

1. Arnold Magnetic Technologies Co., 2014, www.arnoldmagnetic.com (15.06.2014)

2. Gosiewski Z., Ambroziak L., 2012, Formation flight control scheme for unmanned aerial vehicles, Lecture Notes in Control and Information Science, 422, 331-340

3. Gosiewski Z., Cieśluk J., Ambroziak L., 2011, Vision-based obstacle avoidance for Unmanned Aerial Vehicles, 4th International Congress on Image and Signal Processing, P. Qiu, Y. Xiang, Y. Ding, D. Li, L. Wang (Edit.), IEEE, 2053-2058

4. Kolm H., Mongeau P., Williams F., 1980, Electromagnetic launchers, IEEE Transactions on Magnetics, Mag-16, 5, 719-721

5. Kondratiuk M., 2013, Designing and investigating of the magnetic coil launcher with controlled acceleration of the launching object (in Polish), PhD thesis, BUT

6. Kondratiuk M., Gosiewski Z., 2013a, Laboratory stand of an electromagnetic multi-coil launcher for micro aerial vehicles, Mechatronic Systems and Materials IV, Z. Gosiewski, Z. Kulesza (Edit.), Durnten-Zurich, Trans-Tech Publications, Solid State Phenomena, 334-339

7. Kondratiuk M., Gosiewski Z., 2013b, Simulation model of an electromagnetic multi-coil launcher for micro aerial vehicles, Mechatronic Systems and Materials IV, Z. Gosiewski, Z. Kulesza, Durnten-Zurich, Solid State Phenomena, 406-411

8. Kondratiuk M., Gosiewski Z., 2014, Comparison of electromagnetic coil launcher model with real-device characteristics, Solid State Phenomena, 214, 58-66

9. KownACKi C., 2013, Successful application of miniature laser rangefinders in obstacle avoidance method for fixed wing MAV, International Journal of Robotics and Automation, 28, 3, 292-298 
10. Ładyżyńska-Kozdraś E., Falkowski K., Sibilska-Mroziewicz A., 2014a, Physical model of the cart of an UAV catapult using Meissner effect (in Polish), [In:] Mechanika w Lotnictwie, ML-XVI 2014, t. II, K. Sibilski (Edit.), PTMTS Warsaw, 231-241

11. ŁAdyżyńska-Kozdraś E., FAlKowski K., Sibilska-MroziewiCz A., 2014b, Vibration analysis of a levitating cart of a magnetic catapult (in Polish), [In:] Mechanika w Lotnictwie, ML-XVI 2014, t. II, K. Sibilski (Edit.), PTMTS Warsaw, 243-250

12. MCNAB I., 2007, A research program to study airborne launch to space, IEEE Transactions on Magnetics, 43, 1, 486-490

13. Mystkowski A., 2013, Piezo-stack vortex generators for boundary layer control of a delta wing micro-aerial vehicle, Mechanical Systems and Signal Processing, 40, 783-790

14. MystKowski A., 2014, Implementation and investigation of a robust control algorithm for an unmanned micro-aerial vehicle, Robotics and Autonomous Systems, 62, 1187-1196

15. Patterson D., Monti A., Brice C., Dougal R., Pettus R., Srinivas D., Dilipchandra K., Bertoncelli T., 2002, Design and simulation of an electromagnetic aircraft launch system, Industry Applications Conference, 37th IAS Annual Meeting, 1950-1957

16. Perkowski W., 2008, Pneumatic launcher for unmanned aerial vehicles, Transport and Engineering, 27, 181

17. Sibilski K., Falkowski K., Olejnik A., 2014, Magnetic take-off and landing system of airplanes - project GABRIEL (in Polish), [In:] Mechanika w Lotnictwie, ML-XVI 2014, t. II, K. Sibilski (Edit.), PTMTS Warsaw, 216-229

18. Tomczuk B., Schröder G., Waindok A., 2007, Finite element analysis of the magnetic field and electromechanical parameters calculation for a slotted permanent magnet tubular linear motor, IEEE Transactions on Magnetics, 43, 7, 3229-3236

19. Tomczuk B., Waindok A., 2009, Linear motors in mechatronics - achievements and open problems, Transfer of innovation to the interdisciplinary teaching of mechatronics for the advanced technology needs, Opole University of Technology, 343-360

20. Tomczuk B., Waindok A., Wajnert D., 2012, Transients in the electromagnetic actuator with the controlled supplier, Journal of Vibroengineering, 14, 1, 39-44 